\title{
Recent progress on exotic charmonium from BESIII
}

\author{
Aiqiang Guo* \\ (On behalf of the BESIII collaboration) \\ Deutsches Elektronen-Synchrotron, Hamburge, Gemmany \\ Institute of High Energy Physics, Chinese Academy of Sciences, Beijing, China \\ E-mail: guoaqdihep.ac.cn
}

\begin{abstract}
The BESIII experiment is an unique electron-position machine operating at $\tau$-charm energy region. It has collected about $5 \mathrm{fb}^{-1}$ data at centre-mass-energy between 3.8 and $4.6 \mathrm{GeV}$ from 2011 and a series of charged exotic charmomium states, which were named as $Z_{c}$ states, were observed. These observations provide strong evidence that the new type of hadrons beyond meson and baryon exist. Besides, lots of new informations about neutral exotic states, labeled as $\mathrm{XY}$ states, were also observed. These observations include the new production mechanism of $\mathrm{X}(3872)$ and a candidate of $\psi\left(1^{3} D_{2}\right)$. Some new structures were also found from the cross section measurement in several exclusive processes. In this proceeding, we report these progresses from BESIII experiment in detail.
\end{abstract}

XXIV International Workshop on Deep-Inelastic Scattering and Related Subjects

11-15 April, 2016

DESY Hamburg, Germany

${ }^{*}$ Speaker. 


\section{1. introduction}

According to quark model, the most stable hadrons are meson and baryon. But, more types of hadrons, such as tetra-quarks, penta-quarks and glueball, are allowed by QCD. We have found hundreds of meson resonances and dozens of baryons resonances in experiment in the past decades, most of them are proved to be conventional hadrons. The general explanation is that other types of hadrons are probably broad or strongly mixed with conventional mesons and baryons, in which case identifying them experimentally would be difficult.

Recent discoveries of charged heavy quarkonium shatter this explanation. In November 2011, the Belle Collaboration discovered the $Z_{b}^{+}(10610)$ and $Z_{b}^{+}$(10650) [1], whose decays into $\Upsilon \pi$ reveal their constituents to be $b \bar{b} u \bar{d}$. In April 2013, the BESIII and Belle Collaborations discovered the $Z_{c}^{+}(3900)[2,3]$, whose decays into $J / \psi \pi^{+}$reveal its constituents to be $c \bar{c} u \bar{d}$. These charged tetraquark states are relatively narrow although they are above the thresholds for decays into a pair of heavy-light mesons. The discoveries of these manifestly exotic states provide strong evidence that more types of bound states than just mesons and baryons exist.

BESIII is the third generation detector operating on BEPCII, which is an electron-position collider work at $\tau$-charm energy region. The designed luminosity of BEPCII is $1^{33} \mathrm{~cm}^{-2} \mathrm{~s}^{-1}$ and have been achieved after 7 years operating. Since 2011, BESIII has collected about $5 \mathrm{fb}^{-1}$ data at centre-mass-energy between 3.8 and $4.6 \mathrm{GeV}$ and yield many important observations on XYZ physics, which will be introduced in the following sections.

\section{Review of observation of $Z_{c}(3900)$ and $Z_{c}(4020)$}

In the year before 2013, although there are several charged exotic quarkonium are found, but all of them only observed by one experiment. The discovery of $Z_{b}^{+}(10610)$ and $Z_{b}^{+}(10650)$ indicates the possible existence of analogy charged charmonium like states.

In the study of the process $e^{+} e^{-} \rightarrow \pi^{+} \pi^{-} J / \psi$ at $\sqrt{s}=4.260 \mathrm{GeV}$ [2], A charged structure, named as $Z_{c}(3900)$, in the $\pi^{ \pm} J / \psi$ invariant mass spectrum was observed. Meanwhile, a structure around $4.02 \mathrm{GeV}$ was observed in the $\pi^{ \pm} h_{c}$ invariant mass spectrum in the process of $e^{+} e^{-} \rightarrow$ $\pi^{+} \pi^{-} h_{c}$, named as $Z_{c}(4020)$ [4]. After the discovery of charged $Z_{c}(3900)$ and $Z_{c}(4020)$, their iosspin partners, the $Z_{c}(3900)^{0}$ and $Z_{c}(4020)^{0}$, were also observed in events of $e^{+} e^{-} \rightarrow \pi^{0} \pi^{0} J / \psi[5]$ and $e^{+} e^{-} \rightarrow \pi^{0} \pi^{0} h_{c}$ [6]. The measured cross sections of these neutral processes are consistent with the iso-spin conservation.

Since the mass of $Z_{c}(3900)\left(Z_{c}(4020)\right)$ is close to the $D \bar{D}^{*}\left(D^{*} \bar{D}^{*}\right)$ mass threshold, One natural explanation is that $Z_{c}(3900)$ and $Z_{c}(4020)$ state is $S$-wave $D^{*} \bar{D}$ and $D^{*} \bar{D}^{*}$ molecular states or molecular-type resonances. Therefore, study of open charm decay is necessary to validate this assumption.

BESIII experiment performed a study of the process $e^{+} e^{-} \rightarrow \pi^{ \pm}\left(D \bar{D}^{*}\right)^{\mp}$ [7]. In the recoil mass of the bachelor $\pi^{ \pm}$, an enhancement was observed near the $D \bar{D}^{*}$ mass threshold, named $Z_{c}$ (3885). From a study of $e^{+} e^{-} \rightarrow \pi^{ \pm}\left(D^{*} \bar{D}^{*}\right)^{\mp}$ process [8], a structure near the $\left(D^{*} \bar{D}^{*}\right)$ threshold was observed (named as $Z_{c}(4025)$ ) in the recoil mass spectrum of the bachelor $\pi^{ \pm}$. 


\subsection{New informations of $Z_{c}$ states}

Since the $Z_{c}(3900)$ and $Z_{c}(4020)$ are proved to be iso-spin triplet, therefore, the $Z_{c}(3885)$ and $Z_{c}(4025)$ are also should be iso-spin triplet if we assume the $Z_{c}(3900)\left(Z_{c}(4020)\right)$ and $Z_{c}(3885)$ $\left(Z_{c}(4025)\right)$ belong to the same state.

A neutral structure in the $D \bar{D}^{*}$ system around the $D \bar{D}^{*}$ mass threshold, which is denoted as $Z_{c}(3885)^{0}$ is observed in the process $e^{+} e^{-} \rightarrow \pi^{0}\left(D^{+} \bar{D}^{*-}\right)$ and $e^{+} e^{-} \rightarrow \pi^{0}\left(D^{0} \bar{D}^{* 0}\right)$ at $\sqrt{s}=4.23$ and $4.26 \mathrm{GeV}$ [9]. As shown in the left plot of Fig. 1, its pole mass and width are determined to be $\left(3885.7_{-5.7}^{+4.3} \pm 8.4\right) \mathrm{MeV} / \mathrm{c}^{2}$ and $\left(35_{-12}^{+11} \pm 15\right) \mathrm{MeV}$, respectively. The Born cross section are measured to be $(77 \pm 13 \pm 17) \mathrm{pb}$ at $4.23 \mathrm{GeV}$ and $(47 \pm 9 \pm 10)$ bp at $4.26 \mathrm{GeV}$. The ratio of $\frac{B\left(Z_{c}(3885)^{0} \rightarrow D^{+} \bar{D}^{*-}\right)}{B\left(Z_{c}(3885)^{0} \rightarrow D^{0} \bar{D}^{* 0}\right)}$ is determined to be $0.96 \pm 0.18 \pm 0.12$, which is consistent with iso-spin conservation.

A study of the process $e^{+} e^{-} \rightarrow \pi^{0}\left(D^{*} \bar{D}^{*}\right)^{0}$ was performed using $1092 \mathrm{pb}^{-1}$ data taken as $\sqrt{s}=4.23 \mathrm{GeV}$ and $826 \mathrm{pb}^{-1}$ data taken at $\sqrt{s}=4.26 \mathrm{GeV}$ [10]. As shown in right plot of Fig. 1, a neutral structure near the $\left(D^{*} \bar{D}^{*}\right)^{0}$ mass threshold in the $\pi^{0}$ recoil mass spectrum, which we denote as $Z_{c}(4025)^{0}$. Its pole mass and pole width are determined to be $\left(4025.5_{-4.7}^{+2.0} \pm 3.1\right) \mathrm{MeV} / c^{2}$ and $(23.0 \pm 6.0 \pm 1.0) \mathrm{MeV}$, respectively. The Born cross sections of $e^{+} e^{-} \rightarrow Z_{c}(4025)^{0} \pi^{0} \rightarrow$ $\left(D^{*} \bar{D}^{*}\right)^{0} \pi^{0}$ are measured to be $(61.6 \pm 8.2 \pm 9.0) \mathrm{pb}$ at $\sqrt{s}=4.23 \mathrm{GeV}$ and $(43.4 \pm 8.0 \pm 5.4) \mathrm{pb}$ at $\sqrt{s}=4.26 \mathrm{GeV}$.
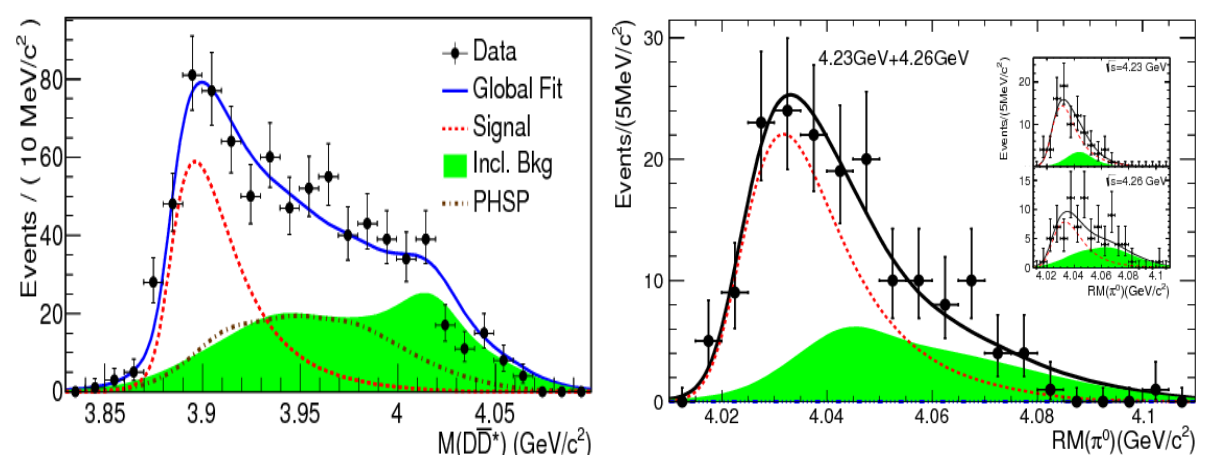

Figure 1: Left: Fit to the $M\left(D \bar{D}^{\star}\right)$ distribution in $e^{+} e^{-} \rightarrow \pi^{0}\left(D \bar{D}^{*}\right)^{0}$ at sqrts $=4.23$ and $4.26 \mathrm{GeV}$. Right: Fit to $\mathrm{RM}\left(\pi^{0}\right)$ in the process $e^{+} e^{-} \rightarrow \pi^{0}\left(D^{*} \bar{D}^{*}\right)^{0}$ at sqrts $=4.23$ and $4.26 \mathrm{GeV}$.

Besides the mass and width, the partial wave analysis also performed to determine the quantum number of $Z_{c}$ states. Using the $1.92 \mathrm{fb}^{-1}$ data taken at $\sqrt{s}=4.23 \mathrm{GeV}$ and $4.26 \mathrm{GeV}$, the spin parity of the $Z_{c}(3900)^{ \pm}$is determined to be $J^{P}=1^{+}$with a statistical significance larger than $7.3 \sigma$ over other quantum number in the partial wave analysis of $e^{+} e^{-} \rightarrow \pi^{+} \pi^{-} J / \psi$. The analysis determines its pole mass $M_{\text {pole }}=(3887.0 \pm 0.8 \pm 10.0) \mathrm{MeV} / \mathrm{c}^{2}$ and pole width $\Gamma_{\text {pole }}=(45.2 \pm$ $4.8 \pm 16.8) \mathrm{MeV} / \mathrm{c}^{2}$.

\section{New observations of $\mathrm{X}$ states}

Currently, X(3872) was only observed in B meson decays or hadron collision. Since its quantum number is $1^{++}$, it could also be produced through radiative transition from the vector charmonium or charmonium-like states. 
The process $e^{+} e^{-} \rightarrow \gamma \pi^{+} \pi^{-} J / \psi$ has been studied at BESIII experiment with the data samples collected at the CM energy $\sqrt{s}=4.009,4.230,4.260$, and $4.360 \mathrm{GeV}$ [11]. Left plot of Fig. 2 shows the $\pi^{+} \pi^{-} J / \psi$ invariant mass spectrum summed over all the energy points and the best fit results. Obvious $\mathrm{X}(3872)$ signals were observed with a statistical significance of $6.3 \sigma$, the mass of the $\mathrm{X}(3872)$ was measured to be $(3872.1 \pm 0.8 \pm 0.3) \mathrm{MeV} / \mathrm{c}^{2}$.
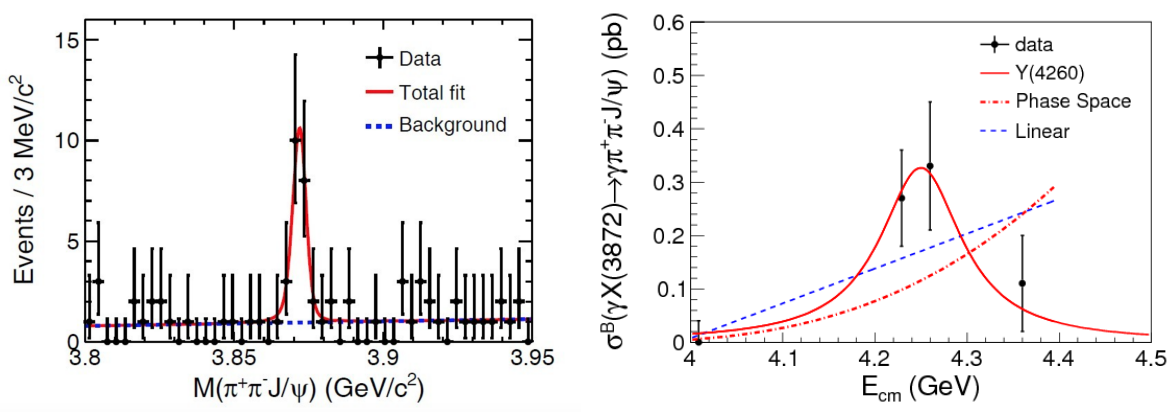

Figure 2: Fit to the summed $\pi^{+} \pi^{-} J / \psi$ invariant mass spectrum. Dots with error bars are data, and the curves are the best fit.

The product of the Born cross section and the branching fraction of $X(3872) \rightarrow \pi^{+} \pi^{-} J / \psi$ as a function of CM energy were shown in right plot of Fig. 2. The measured cross section supports that the $\mathrm{X}(3872)$ come from the radiative transition of $\mathrm{Y}(4260)$.

The lightest charmonium state above the $D \bar{D}$ threshold is the $\psi(3770)$, which is currently identified as the $1^{3} D_{1}$ state. Until now there have been no definitive observations of its two Dwave spin-triplet partner states, i.e., the $1^{3} D_{2}$ and $1^{3} D_{3}$. Recently, the Belle Collaboration reported evidence for a narrow resonance $X(3823) \rightarrow \gamma \chi_{c 1}$ in B meson decays with $3.8 \sigma$ significance [12], and suggested that this is a good candidate for the $1^{3} D_{2}$ charmonium, which is denoted as $\psi_{2}$.

BESIII report a search for the $\psi_{2}$ via the process: $e^{+} e^{-} \rightarrow \pi^{+} \pi^{-} X(3823), X(3823) \rightarrow \gamma \chi_{c 1}$ using $4.67 \mathrm{pb}^{-1}$ data collected at $\sqrt{s}=4.19$ to $4.60 \mathrm{GeV}$ [13]. The $\mathrm{X}(3823)$ is observed with significance of $6.2 \sigma$ and the mass is measured to be $(3821.7 \pm 1.3 \pm 0.7) \mathrm{MeV} / \mathrm{c}^{2}$ (as shown in left plot of Fig. 3). The angular distribution of $\mathrm{X}(3823)$ in the $e^{+} e^{-} \mathrm{CM}$ frame, which is shown in right plot of Fig. 3, support the assumption that X(3823) is D wave charmonium.
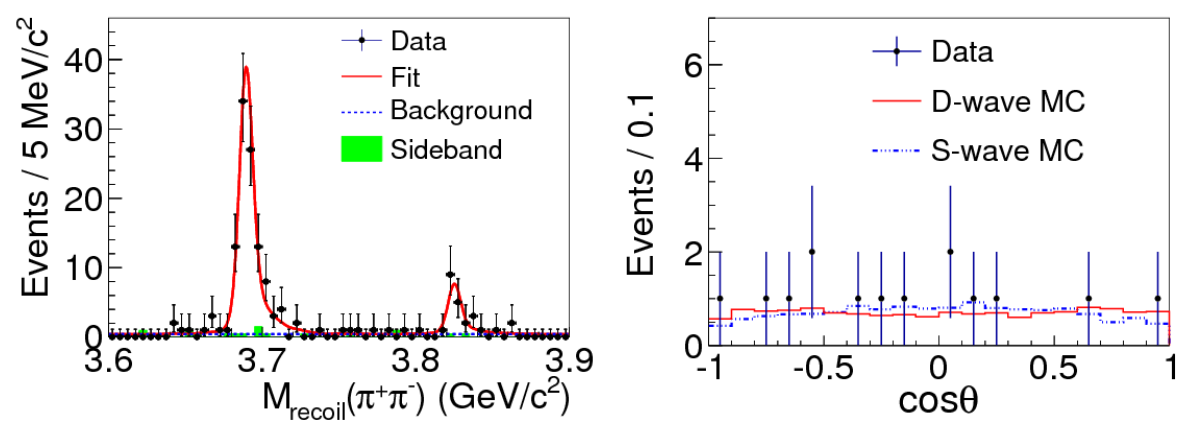

Figure 3: Left: Simultaneous fit to the $M_{\text {recoil }}\left(\pi^{+} \pi^{-}\right)$distribution of $\gamma \chi_{c 1}$ event. Right: The $\mathrm{X}(3823)$ scattering angle distribution. 


\section{New informations of $Y$ states}

BESIII also study a serials exclusive hadronic decays to search for vector exotic states. They should be shown up in the energy dependent cross section if a resonance exist.

The production of $e^{+} e^{-} \rightarrow \omega \chi_{c J}(J=0,1,2)$ was studied at BESIII experiment [14] based on data samples collected at $9 \mathrm{CM}$ energy points from $\sqrt{s}=4.21$ to $4.42 \mathrm{GeV}$ before. $e^{+} e^{-} \rightarrow \omega \chi_{c 0}$ was observed for the first time at 4.23 and $4.26 \mathrm{GeV}$, while signals of $e^{+} e^{-} \rightarrow \omega \chi_{c 1}$ and $\chi_{c 2}$ were not significant. Recently, this process was studied at the data sample taken at $\sqrt{s}>4.4 \mathrm{GeV}$ and clear signal of $e^{+} e^{-} \rightarrow \omega \chi_{c 1,2}$ were observed for the first time [15]. With $1074 \mathrm{pb}^{-1}$ data near $\sqrt{s}=4.42 \mathrm{GeV}$, cross section of $e^{+} e^{-} \rightarrow \omega \chi_{c 2}$ was measured to be $(20.9 \pm 3.2 \pm 2.5) \mathrm{pb}$. with with $567 \mathrm{pb}^{-1}$ data taken at $\sqrt{s}=4.60 \mathrm{GeV}$, the cross section of $e^{+} e^{-} \rightarrow \omega \chi_{c 1}$ was measured to be $(9.5 \pm 2.1 \pm 1.3) \mathrm{pb}$. The energy dependent cross section of $e^{+} e^{-} \rightarrow \omega \chi_{c J}(J=0,1,2)$ are shown in Fig. 4.

The top plot of Fig. 4 shows the cross section of $e^{+} e^{-} \rightarrow \omega \chi_{c 0}$ as a function of CM energy, in which the cross section peaks at $4.23 \mathrm{GeV}$, and that indicates the production of $\omega \chi_{c 0}$ does not arise from Y(4260). The bottom plot of Fig. 4 shows the cross section of $e^{+} e^{-} \rightarrow \omega \chi_{c 2}$, which can be described by BW function of $\psi(4415)$.

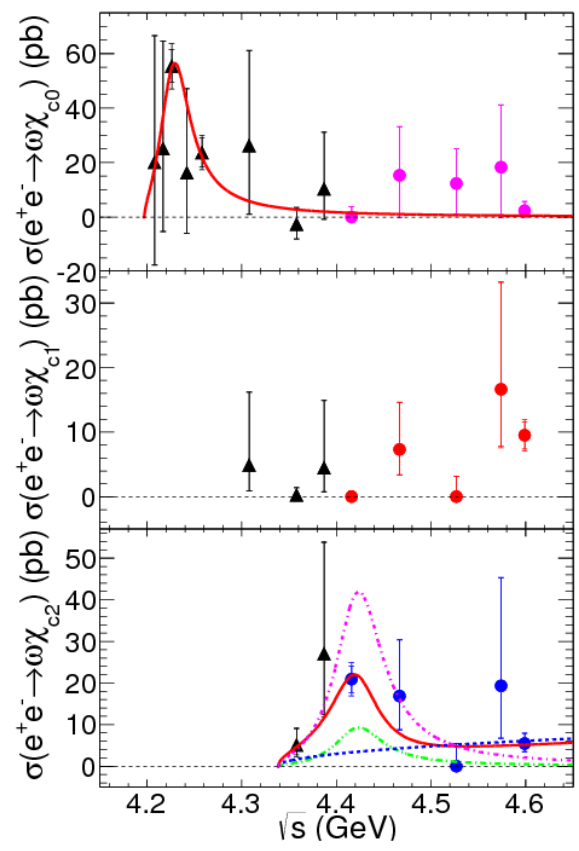

Figure 4: Measured Born cross section for $e^{+} e^{-} \rightarrow \omega \chi_{c J}(\mathrm{~J}=0,1,2)$ as a function of the center of mass energy.

Using data samples collected with the BESIII detector operating at the BEPCII collider at center-of-mass energies from 3.810 to $4.600 \mathrm{GeV}$, we perform a study of $e^{+} e^{-} \rightarrow \eta J / \psi$ and $e^{+} e^{-} \rightarrow \eta^{\prime} J / \psi$. Statistically significant signals of $e^{+} e^{-} \rightarrow \eta J / \psi$ are observed at $\mathrm{s}=4.190,4.210$, $4.220,4.230,4.245,4.260,4.360$ and $4.420 \mathrm{GeV}$ [16]. and Statistically significant signals of $e^{+} e^{-} \rightarrow \eta^{\prime} J / \psi$ are observed at $\mathrm{s}=4.230$, and $4.260 \mathrm{GeV}[17]$. 
The measured energy-dependent Born cross section for $e^{+} e^{-} \rightarrow \eta J / \psi$ and $e^{+} e^{-} \rightarrow \eta^{\prime} J / \psi$ shows in Fig. 5. An enhancement around $4.2 \mathrm{GeV}$ is found in the cross section of $e^{+} e^{-} \rightarrow \eta J / \psi$. The cross section measurement of $e^{+} e^{-} \rightarrow \eta^{\prime} J / \psi$ support the $\psi(4160)$ decay, but we can't draw a clear conclusion due to the low statistics.
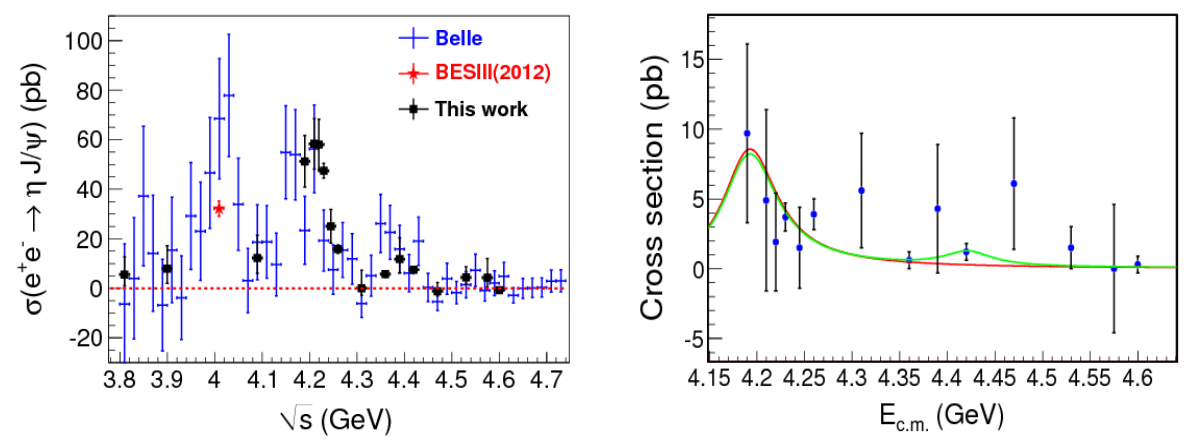

Figure 5: Left: comparison of Born cross section $e^{+} e^{-} \rightarrow \eta J / \psi$. Right:Fit to $\sigma\left(e^{+} e^{-} \rightarrow \eta^{\prime} J / \psi\right)$ with a resonance (solid curve).

\section{Summary}

Dramatic progresses have been made in the charmonium spectroscopy above open charm threshold from BESIII experiment. New class of charged charmonium-like states have been discovered. They provide strong evidence that more types of bound states than just mesons and baryons should exist. In addition, a candidate of $\psi\left(1^{3} D_{2}\right)$ also was observed. The nature of most of XYZ states is not clear so far, more experimental measurements and theory calculations are necessary to solve the puzzle.

\section{References}

[1] A. Bondar et al. [Belle Collaboration], Phys. Rev. Lett. 108, 122001 (2012).

[2] M. Ablikim et al. [BESIII Collaboration], Phys. Rev. Lett. 110, 252001 (2013).

[3] Z. Q. Liu et al. [Belle Collaboration], Phys. Rev. Lett. 110, 252002 (2013).

[4] M. Ablikim et al. [BESIII Collaboration], Phys. Rev. Lett. 111, no. 24, 242001 (2013).

[5] M. Ablikim et al. [BESIII Collaboration], Phys. Rev. Lett. 115, no. 11, 112003 (2015) doi:10.1103/PhysRevLett.115.112003.

[6] M. Ablikim et al. [BESIII Collaboration], Phys. Rev. Lett. 113, no. 21, 212002 (2014).

[7] M. Ablikim et al. [BESIII Collaboration], Phys. Rev. Lett. 112, no. 2, 022001 (2014).

[8] M. Ablikim et al. [BESIII Collaboration], Phys. Rev. Lett. 112, no. 13, 132001 (2014).

[9] M. Ablikim et al. [BESIII Collaboration], Phys. Rev. Lett. 115 (2015) no.22, 222002 doi:10.1103/PhysRevLett.115.222002.

[10] M. Ablikim et al. [BESIII Collaboration], Phys. Rev. Lett. 115, no. 18, 182002 (2015) doi:10.1103/PhysRevLett.115.182002. 
[11] M. Ablikim et al. [BESIII Collaboration], Phys. Rev. Lett. 112, no. 9, 092001 (2014).

[12] V. Bhardwaj et al. [Belle Collaboration], Phys. Rev. Lett. 111, no. 3, 032001 (2013) doi:10.1103/PhysRevLett.111.032001.

[13] M. Ablikim et al. [BESIII Collaboration], Phys. Rev. Lett. 115, no. 1, 011803 (2015) doi:10.1103/PhysRevLett.115.011803.

[14] M. Ablikim et al. [BESIII Collaboration], Phys. Rev. Lett. 114, no. 9, 092003 (2015) doi:10.1103/PhysRevLett.114.092003.

[15] M. Ablikim et al. [BESIII Collaboration], Phys. Rev. D 93, no. 1, 011102 (2016) doi:10.1103/PhysRevD.93.011102.

[16] M. Ablikim et al. [BESIII Collaboration], Phys. Rev. D 91, no. 11, 112005 (2015) doi:10.1103/PhysRevD.91.112005.

[17] M. Ablikim et al. [BESIII Collaboration], [arXiv:1605.03256 [hep-ex]]. 